\title{
Sofrimento psíquico entre os profissionais de enfermagem durante a pandemia da COVID-19: Scoping Review
}

\author{
Psychological distress among nursing professionals during the COVID-19 pandemic: Scoping Review \\ Distrés Psicológico entre los profesionales de enfermería durante la pandemia de COVID-19: Scoping \\ Review
}

Fernanda Berchelli Girão Miranda ${ }^{1}$ (]) Mellina Yamamura ${ }^{1}$ (1) Sarah Salvador Pereira ${ }^{1}$ (1) Caroline dos Santos Pereira ${ }^{1}$ (D) Simone Teresinha Protti-Zanatta ${ }^{1}$ (1) Marceli Karina Costa $^{1}$ (1) Sonia Regina Zerbetto ${ }^{1}$ (D)

1. Universidade Federal de São Carlos, Programa de Pós-Graduação em Enfermagem. São Carlos, SP. Brasil.
Autor correspondente:

Fernanda Berchelli Girão Miranda. E-mail: fernanda.berchelli@ufscar.br.

Recebido em 04/09/2020.

Aprovado em 21/12/2020.

DOI:https://doi.org/10.1590/2177-9465-EAN-2020-0363

\begin{abstract}
Resumo
Objetivo: mapear sistematicamente a produção de conhecimento, com a literatura nacional e internacional, de situações de sofrimento psíquico que os profissionais de enfermagem vivenciam quando expostos à pandemia da COVID-19. Método: Scoping Review conforme Joanna Briggs Institute Reviewer's Manual for Scoping Reviews e PRISMA-ScR. Executaram-se buscas nas bases eletrônicas entre abril e junho de 2020, por intermédio dos descritores. Revisores aos pares analisaram a relevância dos estudos, selecionando os que responderam à pergunta de investigação. Resultados: selecionaram-se 38 estudos. As situações de sofrimento psíquico mais relatadas relacionaram-se à sobrecarga de trabalho, escassez ou ausência de equipamento de proteção individual, medo de se infectar, infectar outras pessoas e estar na linha de frente junto a pacientes com diagnóstico ou suspeita de COVID-19. Os sinais e sintomas de sofrimento psíquico mais encontrados foram ansiedade, depressão, insônia, estresse, estresse pós-traumático e medo. Conclusões e implicações para a prática: os profissionais de enfermagem enfrentam situações de sofrimento psíquico, principalmente desencadeadas por fatores relacionados às condições de trabalho, manifestando sintomas depressivos, de ansiedade e de estresse, que podem permanecer por longo período. As instituições de saúde precisaram implementar ações de capacitação, proteção e segurança, bem como suporte e apoio psicossocial em curto espaço de tempo.
\end{abstract}

Palavras-chave: Profissionais de Enfermagem; Equipe de Enfermagem; Sofrimento Psíquico; Saúde Mental; COVID-19.

\begin{abstract}
Objective: To design a systematic map of the production of knowledge, together with national and international literature, on situations of psychological distress that nursing professionals experience when exposed to the COVID-19 pandemic. Method: Scoping Review according to Joanna Briggs Institute Reviewer's Manual for Scoping Reviews and PRISMA-ScR. We held searches between April and June 2020 in the electronic databases by means of descriptors; the peer reviewers analyzed the relevance of the studies, selecting those that answered the research question. Results: We selected 38 studies. The most reported situations of psychological distress were related to work overload, scarcity or absence of individual protective equipment, fear of becoming infected, infecting other people and being in the front line with patients diagnosed or suspected for COVID-19. The most common signs and symptoms of psychological distress were: anxiety, depression, insomnia, stress, post-traumatic stress and fear. Conclusions and implications for practice: Nursing professionals face situations of psychological distress, mainly triggered by factors related to work conditions, manifesting depressive symptoms, anxiety and stress, which may remain for a long period. Health institutions will need to implement training, protection and security actions, as well as psychosocial help and support in a short period of time.
\end{abstract}

Keywords: Nurse Practitioners; Nursing, Team; Stress, Psychological; Mental Health; COVID-19.

\section{Resumen}

Objetivo: Mapear sistemáticamente la producción de conocimiento, junto con la literatura nacional e internacional, de las situaciones de distrés psicológico experimentadas por los profesionales de enfermería cuando se exponen a la pandemia de COVID-19. Método: Scoping Review según el Joanna Briggs Institute Reviewer's Manual for Scoping Reviews y el PRISMA-ScR. Las búsquedas tuvieron lugar entre abril y junio de 2020 en las bases de datos electrónicas mediante descriptores; los revisores analizaron la relevancia de los estudios, seleccionando aquellos que respondían a la pregunta de investigación. Resultados: Se seleccionaron 38 estudios. Las situaciones de distrés psicológico más informadas estaban relacionadas con la sobrecarga de trabajo, la escasez o ausencia de equipos de protección personal, el miedo a infectarse, a infectar a otras personas y a estar en primera línea con pacientes diagnosticados o sospechosos de COVID-19. Los signos y síntomas más comunes de distrés psicológico eran: ansiedad, depresión, insomnio, estrés, estrés postraumático y miedo. Conclusiones e implicaciones para la práctica: Los profesionales de enfermería afrontan situaciones de distrés psicológico, desencadenadas principalmente por factores relacionados con las condiciones de trabajo, manifestando síntomas depresivos, ansiedad y estrés, que pueden permanecer durante un largo período. Las instituciones sanitarias necesitarán aplicar medidas de capacitación, protección y seguridad, así como de soporte y apoyo psicosocial en un breve período de tiempo.

Palabras clave: Enfermeras Practicantes; Grupo de Enfermería; Estrés Psicológico; Salud Mental; COVID-19. 


\section{INTRODUÇÃO}

O mundo vem enfrentando uma pandemia denominada COVID-19 (Coronavirus Disease 2019), que envolve um novo coronavírus (2019-nCoV), o qual desencadeia a Síndrome Respiratória Aguda Grave (SARS-CoV-2). ${ }^{1}$ Devido à evolução da doença na China, a taxa de mortalidade foi estimada entre 2 e 3\%, ${ }^{1}$ e no início de março de 2020, 3,4\%. ${ }^{2}$ Diante dessa situação pandêmica e crítica, os profissionais de saúde - e mais especificamente os profissionais de enfermagem - estão na linha de frente do atendimento e cuidado às pessoas com COVID-19, o que pode desencadear sofrimento psíquico. ${ }^{3,4}$

Estudos chineses e nacionais apontam que profissionais de saúde - principalmente enfermeiros - expostos à doença COVID-19, durante o período da epidemia, sofreram impactos negativos na saúde mental $^{3-6}$ relacionados ao contexto de trabalho. ${ }^{3,5}$ Entretanto, o sofrimento psíquico repercute e impacta a vida do profissional de enfermagem no âmbito psicossocial e do seu bem-estar geral, o que denota a relevância de identificar tais situações de sofrimento mental. Neste estudo, o conceito de sofrimento psíquico não se limita à identificação de sinais e sintomas do transtorno mental, mas também às situações evidenciadas entre o profissional de enfermagem em sua "existência-sofrimento na relação com o corpo social". ${ }^{7}$ Nessa perspectiva, o sofrimento do profissional de enfermagem associa-se às diversas relações deste no contexto laboral, social e familiar durante a pandemia. A descoberta de tais condições contribui para que tanto as instituições de saúde como as equipes de enfermagem possam identificar e implementar estratégias de enfrentamento nas dimensões de promoção, tratamento e reabilitação psicossocial. Nesse sentido, para apoiar tal processo, este estudo tem como objetivo mapear sistematicamente, com a literatura nacional e internacional, as situações de sofrimento psíquico que os profissionais de enfermagem vivenciam quando expostos à pandemia da COVID-19.

\section{MÉTODO}

Essa Scoping Review foi conduzida usando a estrutura metodológica desenvolvida conforme o Joanna Briggs Institute Reviewer's Manual for Scoping Reviews ${ }^{8}$ e orientações do PRISMA-ScR. ${ }^{9} \mathrm{O}$ desenvolvimento de Scoping Review é indicado para ser precursor de uma revisão sistemática, identificar os tipos de evidências disponíveis em um determinado campo, analisar lacunas de conhecimento, examinar como a pesquisa é realizada sobre algum tópico ou campo, identificar e esclarecer as principais características ou fatores relacionados a um conceito/ definições na literatura. ${ }^{10}$

Apresenta-se como um método útil para examinar evidências emergentes sobre determinado assunto. ${ }^{11}$ Quanto à construção da pergunta da pesquisa, aplicou-se a estratégia PCC, que representa uma mnemônica relativa a População, Conceito e Contexto, ${ }^{11}$ definindo: P - profissionais de enfermagem; Csofrimento psíquico; C- pandemia. Com relação à busca e seleção dos estudos, estabeleceu-se a seguinte questão norteadora:
"Quais são as situações de sofrimento psíquico vivenciadas pelos profissionais de enfermagem quando expostos à pandemia da COVID-19?".

Com o objetivo de identificar documentos potencialmente relevantes, a busca foi realizada em pares, conforme critérios do $\mathrm{JBI}^{7}$ nas bases de dados Literatura Latino-Americana e do Caribe em Ciências da Saúde (LILACS), National Library of Medicine (PubMed), SCOPUS; na plataforma Web of Science e Periódico Capes, por meio dos descritores e/ou seus sinônimos. Dessa forma, utilizaram-se, de acordo com os Descritores em Ciências da Saúde (DeCS) para cada item da estratégia População $(P)$ : "Profissionais de Enfermagem" OR "Recursos Humanos de Enfermagem" OR "Equipe de Enfermagem" OR Enfermeiros OR Enfermagem AND Conceito (C): "Estresse Psicológico" OR "Agente de Estresse Psicológico" OR "Fatores de Estresse Psicológico" OR "Sofrimento Mental" OR "Sofrimento Psíquico" OR Sofrimentos OR "Transtornos Mentais" OR "Doença Mental" OR "Transtorno Mental" OR "Saúde Mental" AND Contexto (C): "pandemias" OR "pandemia" OR "Infecções por Coronavírus" OR "COVID-19" OR "Doença pelo Novo Coronavírus (2019-nCoV)" OR "Doença por Coronavírus 2019-nCoV" OR "Doença por Novo Coronavírus (2019-nCoV)". Quanto aos descritores do Medical Subject Headings (MeSH), utilizou-se a combinação para cada item da estratégia da População $(P)$ : "Nurse Practitioners" OR "Nursing Staff" OR "Nursing, Team" OR "Nurse" OR "Nursing" AND Conceito (C): "Stress, Psychological" OR "Stress, Psychological" OR "Psychological Stresses" OR "Stresses, Psychological" OR "Stress, Psychologic" OR "Psychologic Stress" OR "Mental Suffering" OR "Suffering, Mental" OR "Mental Disorders" OR "Mental Health" AND Contexto (C): "pandemics" OR "COVID-19" OR "COVID19" OR "2019-nCoV infection" OR "coronavirus disease 2019" OR "COVID-19 pandemic" OR "2019-nCoV disease" OR "2019 novel coronavirus disease" OR "2019 novel coronavirus infection" OR "coronavirus disease-19".

Para a combinação dos descritores, consideraram-se os operadores booleanos AND e OR. Após a realização da busca, incluíram-se as pesquisas realizadas nos idiomas inglês, espanhol e português, com abordagem quantitativa, qualitativa, métodos mistos, estudos primários, revisões sistemáticas, metanálises e/ou metassínteses, livros e guidelines, consensos, editoriais, publicados em fontes indexadas que respondessem à pergunta estabelecida. Não foram incluídos os artigos em idiomas diferentes dos estabelecidos, justificado por falta de domínio da língua pelos autores e financiamento do estudo; literatura cinzenta por se tratar de registros científicos recentes com publicações atuais disponíveis em mídias de bases de dados digitais e devido ao fato de isolamento social no país; publicações de opiniões, retrações, websites e propagandas veiculadas em mídias por não se tratarem de material com rigor científico.

As buscas foram executadas entre os meses de abril e junho de 2020, período no qual todas as publicações foram acessadas, sem quaisquer restrições quanto ao espaço temporal. Para o processo de seleção dos estudos, os títulos e resumos foram analisados por dois revisores independentes. Quando tiveram 
dúvidas sobre a relevância de um estudo baseado em seu resumo, a versão completa do texto foi selecionada para ser analisada. Aleatoriamente, os artigos na íntegra foram distribuídos entre todos os revisores, e novamente dois revisores examinaram independentemente a versão completa do texto dos artigos, a fim de verificar se preenchiam os critérios de inclusão e se estes respondiam à questão norteadora desta revisão. No caso de desacordos entre os pares de revisores, um terceiro profissional resolvia. Os revisores de todas as etapas foram nomeados como autores deste manuscrito.

Para a extração do conteúdo, aplicou-se um instrumento estruturado pelos próprios autores, considerando as seguintes variáveis: base de estudo em que foi identificado o manuscrito; origem do artigo; delineamento do estudo; amostra; local de pesquisa; ferramentas utilizadas para avaliar o sofrimento psíquico; situações de sofrimento psíquico; sinais e sintomas de sofrimento psíquico; e outras situações pertinentes à temática entre os estudos selecionados. Os revisores mapearam os dados independentemente, discutiram os resultados e atualizaram continuamente o formulário de mapeamento de dados em um processo interativo, conforme recomendações do JBI. ${ }^{8}$ As evidências encontradas neste estudo são apresentadas a seguir, de maneira narrativa, por meio de quadros e diagrama. ${ }^{9}$

\section{RESULTADOS}

A pesquisa identificou, nas bases de dados selecionadas, 2.416 registros potencialmente relevantes; 3 foram identificados como duplicados, restando 90 para leitura. Do total de 90 artigos, selecionaram-se apenas 36 para leitura na íntegra, e outros 9, por intermédio das suas referências. Assim, 45 artigos foram incluídos para análise de texto completo por revisores independentes. Após a leitura, outros cinco estudos foram excluídos por não envolverem enfermeiros, um estudo por estar redigido na íntegra da língua francesa e um estudo por estar na íntegra no preprint e sem avaliação de revisores. No fim, 38 estudos foram selecionados por responderem à questão da pesquisa. Para a apresentação dos resultados, numeraram-se as pesquisas de 1 a 38. A descrição detalhada do processo de seleção e inclusão dos artigos encontra-se descrita na Figura 1.

Os artigos incluídos na amostra foram todos publicados no ano de 2020. A maior parte dos estudos encontrados foi publicada na base de dados PubMed, em um total de 23 (60,5\%), seguida por $9(24,0 \%)$ de bibliografia secundária, $5(13,0 \%)$ na SCOPUS e $1(2,5 \%)$ na Web of Science. O Quadro 1 apresenta os estudos analisados segundo o país de origem, o objetivo, a amostra e a abordagem metodológica.

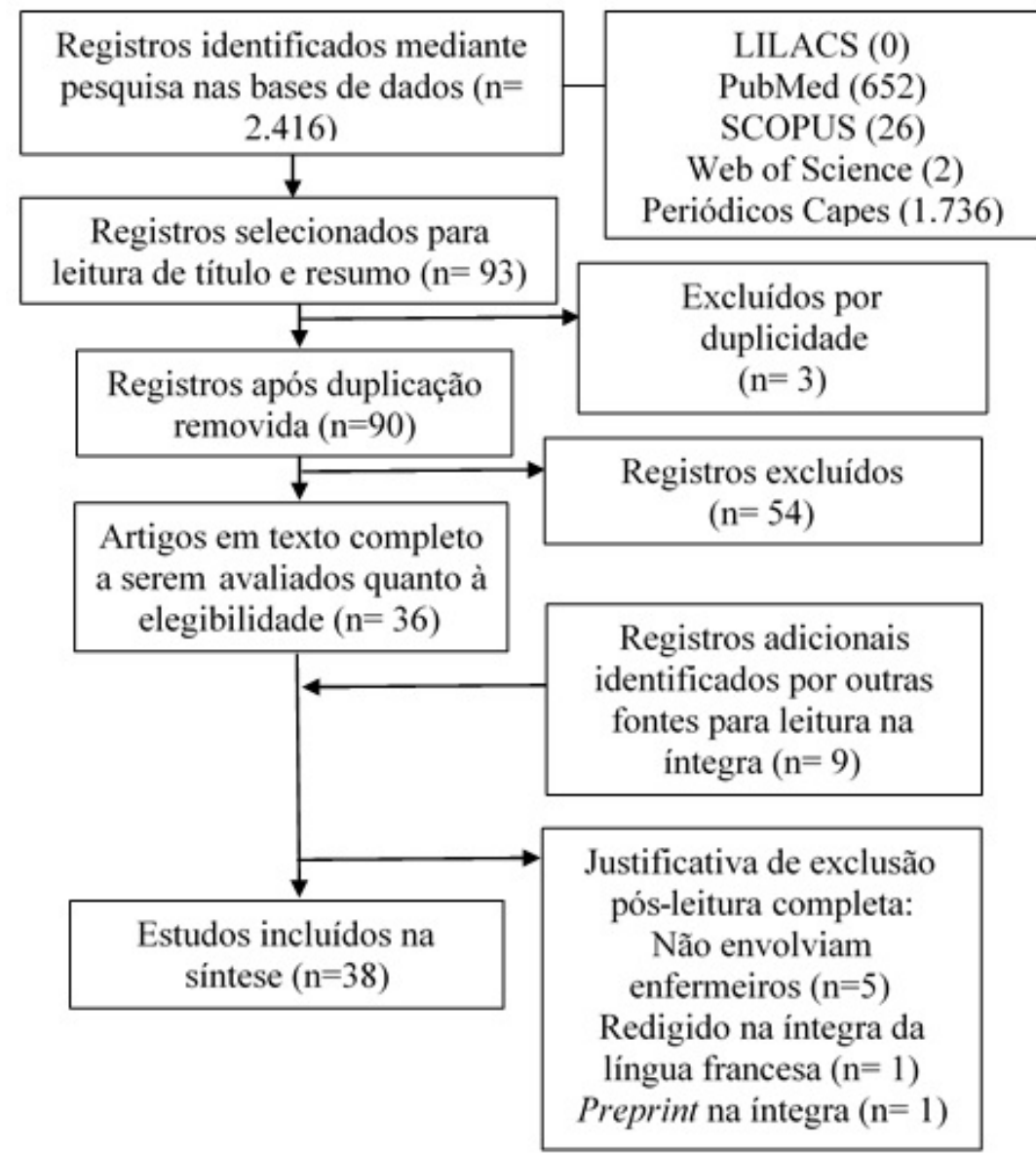

Figura 1. Descrição do processo de seleção dos estudos. São Carlos, SP, Brasil, 2020. Fonte: Elaborada pelos autores 
Quadro 1. Estudos analisados segundo o país de origem, abordagem metodológica e amostra. São Carlos, SP, Brasil, 2020.

\begin{tabular}{|c|c|}
\hline Itens de análise & Estudos \\
\hline \multicolumn{2}{|l|}{ Origem do artigo } \\
\hline Ásia Oriental & $\begin{array}{l}\text { China E1* } \text { E2, E3, E4, E5, E6, E7, E8, E9, E10, E11, }{ }^{12-22} \text { E14, }{ }^{25} \text { E18, E19, }{ }^{29,30} \text { E23, } \\
\text { E24, }{ }^{34,35} \text { E30, E31, E32, }{ }^{41-43} \text { E34, }{ }^{45} \text { E36, }{ }^{47} \text { E37; }{ }^{48} \text { Hong Kong E35 }{ }^{46}\end{array}$ \\
\hline Oriente Médio & Líbano E28 $8^{39}$ \\
\hline Sudeste Asiático & Singapura $\mathrm{E} 12,,^{23} \mathrm{E} 27^{38}$ \\
\hline Subcontinente Indiano & Índia $\mathrm{E} 15,{ }^{26} \mathrm{E} 17 ;{ }^{28}$ Paquistão E21 ${ }^{32}$ \\
\hline Península Arábica & Arábia Saudita E20 31 \\
\hline Europa Ocidental & Itália E22;33 Espanha E29; ${ }^{40}$ Reino Unido E33; ${ }^{44}$ Inglaterra E13 ${ }^{24}$ \\
\hline América do Norte & Estados Unidos E16 27 \\
\hline Pacífico Ocidental & Austrália E25, E26 $6^{36,37}$ \\
\hline Não menciona & $\mathrm{E} 38^{49}$ \\
\hline \multicolumn{2}{|l|}{ Tipo de estudo } \\
\hline Transversal & $\begin{array}{l}\mathrm{E} 2,{ }^{13} \mathrm{E} 6,{ }^{17} \mathrm{E} 7,{ }^{18} \mathrm{E} 9{ }^{20}{ }^{20} \mathrm{E} 11,{ }^{22} \mathrm{E} 12,{ }^{23} \mathrm{E} 18, \mathrm{E} 19, \mathrm{E} 20,{ }^{29-31} \mathrm{E} 22, \mathrm{E} 23, \mathrm{E} 24,{ }^{33-35} \mathrm{E} 27,{ }^{38} \mathrm{E} 30,{ }^{41} \\
\mathrm{E} 31,{ }^{42} \mathrm{E} 34,{ }^{45} \mathrm{E} 36^{47}\end{array}$ \\
\hline Descritivo & $\mathrm{E}^{14}$ \\
\hline Revisões narrativas & $\mathrm{E} 1,{ }^{12} \mathrm{E} 14,{ }^{25} \mathrm{E} 15,{ }^{26} \mathrm{E} 29,{ }^{40} \mathrm{E} 37, \mathrm{E} 38^{48,49}$ \\
\hline Revisões sistemáticas e metanálises & $\mathrm{E} 13,^{24} 4 \mathrm{E} 25^{36}$ \\
\hline Editoriais, comentários ou discussões & $\mathrm{E} 4,{ }^{15} \mathrm{E} 5,{ }^{16} \mathrm{E} 17,{ }^{28} \mathrm{E} 2,{ }^{32} \mathrm{E} 26,{ }^{37} \mathrm{E} 32, \mathrm{E} 33,{ }^{43,44} \mathrm{E} 35^{46}$ \\
\hline Relato de experiência & $\mathrm{E} 16^{27}$ \\
\hline Qualitativos & $\mathrm{E} 10,{ }^{21} \mathrm{E} 28^{39}$ \\
\hline Misto & $\mathrm{E}^{19}$ \\
\hline \multicolumn{2}{|l|}{ Amostra } \\
\hline Estudos com 50 ou menos enfermeiros & $\mathrm{E} 8,{ }^{19} \mathrm{E} 9,{ }^{20} \mathrm{E} 10,{ }^{21} \mathrm{E} 28^{39}$ \\
\hline Estudos com 100 ou mais enfermeiros & $\mathrm{E} 7,{ }^{18} \mathrm{E} 11,{ }^{22} \mathrm{E} 18,{ }^{29} \mathrm{E} 22,{ }^{33} \mathrm{E} 31,{ }^{42} \mathrm{E} 36^{47}$ \\
\hline Estudos com mais de 300 enfermeiros & $\mathrm{E} 20,{ }^{31} \mathrm{E} 24,{ }^{35} \mathrm{E} 27,,^{38} \mathrm{E} 34^{45}$ \\
\hline Estudos com mais de 500 enfermeiros & $\mathrm{E} 2,{ }^{13} \mathrm{E} 3,{ }^{14} \mathrm{E} 6,{ }^{17} \mathrm{E} 11,,^{22} \mathrm{E} 19,{ }^{30} \mathrm{E} 23,{ }^{34} \mathrm{E} 30^{41}$ \\
\hline $\begin{array}{l}\text { Envolvem apenas estudos ou não se } \\
\text { aplica }\end{array}$ & $\begin{array}{l}E 1,{ }^{12} E 4,{ }^{15} E 5,{ }^{16} E 13, E 14, E 15, E 16, E 17,{ }^{24-28} E 21,{ }^{32} E 25, E 26,{ }^{36-40} E 29,{ }^{40} E 32,{ }^{43} E 33,{ }^{44} \\
E 35,{ }^{46} E 37, E 38^{48,49}\end{array}$ \\
\hline
\end{tabular}

*E1=Estudo 1

Fonte: Informações extraídas dos artigos científicos indexados nas bases de dados National Library of Medicine (PubMed), SCOPUS; na plataforma Web of Science, 2020

Dos 38 artigos considerados, 21 (55,3\%) foram realizados na China, 3 (7,9\%) na Índia, 2 (5,3\%) não descreveram o local do estudo ou mencionaram algum país de origem da pesquisa, 2 (5,3\%) na Europa (Itália e Espanha), 2 (5,3\%) no Reino Unido e Inglaterra, $2(5,3 \%)$ em Singapura, $2(5,3 \%)$ na Austrália, 1 $(2,6 \%)$ nos Estados Unidos, 1 (2,6\%) no Paquistão, 1 (2,6\%) no Líbano, 1 (2,6\%) em Hong Kong e 1 (2,6\%) na Arábia Saudita.

Quanto ao tipo de estudo, 17 (44,7\%) tratavam-se de estudos transversais, $7(18,4 \%)$ revisões narrativas, $5(13,2 \%)$ editoriais, $2(5,3 \%)$ comentários ou discussões, $2(5,3 \%)$ qualitativos, 2 $(5,3 \%)$ revisões sistemáticas e metanálises, $1(2,6 \%)$ descritivo, $1(2,6 \%)$ relato de experiência e $1(2,6 \%)$ de abordagem mista.
Considerando a população (amostra) dos estudos do tipo transversal, os enfermeiros e médicos foram os profissionais de maior frequência de investigação. A título elucidativo, a China realizou 13 dos 17 artigos, totalizando cerca de 13.728 enfermeiros e 8.065 médicos, não sendo possível analisar quais profissionais participaram repetidamente das pesquisas.

Em relação à instituição de trabalho dos enfermeiros, 26 $(68,4 \%)$ estudos se relacionaram ao atendimento hospitalar, e os demais - $12(31,6 \%)$ estudos - não especificaram. Os estudos apresentaram as seguintes ferramentas para avaliar o sofrimento psíquico: escalas ( $n=17 ; 36,2 \%)$; aplicativos ( $n=2 ; 4,2 \%$ ); entrevistas ( $n=3 ; 6,4 \%)$ instrumentos ( $n=3 ; 6,4 \%)$, questionários 
( $n=8 ; 17,0 \%)$; e $n=17(29,8 \%)$ não utilizaram nenhum tipo de ferramenta por não se aplicar.

Relacionadas às situações que contribuíram para o sofrimento psíquico, as principais causas associadas encontradas foram: falta de descanso laboral; sobrecarga de trabalho; longa jornada de trabalho; déficit de membros na equipe; Burnout; pressão psicológica; falta de apoio do empregador; baixa satisfação no trabalho. Tais situações foram evidenciadas nos seguintes estudos: E1, ${ }^{12} \mathrm{E} 4,{ }^{15} \mathrm{E} 7, \mathrm{E} 8,{ }^{18,19} \mathrm{E} 12,{ }^{23} \mathrm{E} 14, \mathrm{E} 15, \mathrm{E} 16,{ }^{25-27} \mathrm{E} 19,{ }^{30}$ $\mathrm{E} 21, \mathrm{E} 22,{ }^{32,33} \mathrm{E} 25,{ }^{36} \mathrm{E} 29,{ }^{40} \mathrm{E} 36, \mathrm{E} 37 .{ }^{47,48}$

A segunda situação contribuinte de maior frequência foi a atuação na linha de frente, no cuidado ao paciente com COVID-19, trabalho em ambientes clínicos de alto risco e risco de infecção, representados pelos estudos E2, E3, E4, E5, ${ }^{13-16} \mathrm{E} 13, \mathrm{E} 14,{ }^{24,25}$ $\mathrm{E} 17,{ }^{28} \mathrm{E} 19,{ }^{30} \mathrm{E} 2,{ }^{13} \mathrm{E} 24, \mathrm{E} 25,{ }^{35,36} \mathrm{E} 36,{ }^{47} \mathrm{E} 38 .{ }^{49}$ O medo de contágio próprio e preocupação com a família, amigos, vizinhos ou colegas se caracterizaram uma constante nos estudos $\mathrm{E} 1,{ }^{12} \mathrm{E} 4,{ }^{15} \mathrm{E} 6,{ }^{17}$ $\mathrm{E} 8,{ }^{19} \mathrm{E} 10, \mathrm{E} 11,{ }^{21,22} \mathrm{E} 14,{ }^{25} \mathrm{E} 16, \mathrm{E} 17,{ }^{27,28} \mathrm{E} 20,{ }^{31} \mathrm{E} 25, \mathrm{E} 26, \mathrm{E} 27,{ }^{26-28}$ E37, E38.48,49 Por fim, não diferente do esperado as situações de escassez de Equipamentos de Proteção Individual (EPIs) e assistência ao paciente com limitação de recursos, as quais também apresentaram alta representatividade em E3, E4, ${ }^{14,15}$
$\mathrm{E} 10,{ }^{21} \mathrm{E} 14, \mathrm{E} 15,{ }^{25,26} \mathrm{E} 17,{ }^{28} \mathrm{E} 19,{ }^{30} \mathrm{E} 24,{ }^{35} \mathrm{E} 26,{ }^{37} \mathrm{E} 33 .{ }^{44}$ A seguir, o Quadro 2 contém os sinais e sintomas de sofrimento psíquico apresentados pelos profissionais de enfermagem.

\section{DISCUSSÃO}

Este estudo objetivou identificar quais situações de sofrimento psíquico os profissionais de enfermagem vivenciam quando expostos à pandemia da COVID-19, de acordo com um mapeamento sistemático, contribuindo também na identificação de lacunas existentes no conhecimento mediante a literatura científica nacional e internacional.

Ressalta-se que a complexidade do cuidar humano envolve uma assistência de enfermagem que demanda técnica, ciência, conhecimento, sentimentos e relação humana. Os profissionais vivenciam constantemente situações de dor, sofrimento, morte e perdas, às quais se somam as condições desfavoráveis de trabalho e baixa remuneração. ${ }^{50}$ Para o autor, ${ }^{51}$ nenhum outro profissional realiza o serviço exclusivo dos enfermeiros, sendo impossível igualá-los. Tal complexidade tornou-se ainda mais intensa com o advento da pandemia da COVID-19, pois, além de toda essa carga já enfrentada anteriormente, os enfermeiros

Quadro 2. Estudos analisados segundo sinais e sintomas de sofrimento psíquico. São Carlos, SP, Brasil, 2020.

\begin{tabular}{|c|c|}
\hline Sinais e sintomas de sofrimento psíquico & Estudos \\
\hline Ansiedade & $\begin{array}{l}E 2,{ }^{13} E 5, E 6, E 7,{ }^{16-18} E 10, E 11,{ }^{21,22} E 13, E 14, E 15, E 16, E 17, E 18, E 19, \\
E 20, E 21,{ }^{24-32} E 24,{ }^{35} E 30, E 31,{ }^{41,42} E 35,{ }^{46}{ }^{2} 36^{47}\end{array}$ \\
\hline Sintomas de depressão & $\begin{array}{l}\mathrm{E} 2,^{13} \mathrm{E} 5, \mathrm{E} 6,{ }^{16,17} \mathrm{E}^{20}{ }^{20} \mathrm{E} 11,{ }^{22} \mathrm{E} 13, \mathrm{E} 14,{ }^{24,25} \mathrm{E} 16, \mathrm{E} 17, \mathrm{E} 18,{ }^{27-29} \mathrm{E} 21,{ }^{32} \mathrm{E} 23, \\
\mathrm{E} 24,{ }^{34,35} \mathrm{E} 31,{ }^{42} \mathrm{E} 35^{46}\end{array}$ \\
\hline Insônia & $\mathrm{E} 2,^{13} \mathrm{E} 6,{ }^{17} \mathrm{E} 8,{ }^{19} \mathrm{E} 11, \mathrm{E} 12, \mathrm{E} 13, \mathrm{E} 14,{ }^{22-25} \mathrm{E} 17,{ }^{28} \mathrm{E} 22,{ }^{33} \mathrm{E} 37^{48}$ \\
\hline Estresse & E7, E8, $,^{18,19} \mathrm{E} 11, \mathrm{E} 12,{ }^{22,23} \mathrm{E} 14, \mathrm{E} 15,,^{25,26} \mathrm{E} 17,{ }^{28} \mathrm{E} 20,{ }^{31} \mathrm{E} 25,{ }^{36} \mathrm{E} 29,{ }^{30} \mathrm{E}^{3} 5^{46}$ \\
\hline Sintomas de estresse pós-traumático & $\mathrm{E} 5,{ }^{16} \mathrm{E} 14,{ }^{25} \mathrm{E} 21,{ }^{32} \mathrm{E} 23,{ }^{34} \mathrm{E} 25,{ }^{36} \mathrm{E} 27,{ }^{38} \mathrm{E} 35,{ }^{46} \mathrm{E} 37^{48}$ \\
\hline Medo & $E 5,{ }^{16} \mathrm{E} 10,{ }^{21} \mathrm{E} 16,{ }^{27} \mathrm{E} 20, \mathrm{E} 21,{ }^{31,32} \mathrm{E} 28, \mathrm{E} 29,{ }^{39,40} \mathrm{E} 33^{44}$ \\
\hline Esgotamento físico e mental & $\mathrm{E} 1,{ }^{12} \mathrm{E} 8,{ }^{19} \mathrm{E} 10,{ }^{21} \mathrm{E} 26,{ }^{37} \mathrm{E}^{4} 5^{46}$ \\
\hline Angústia & $\mathrm{E} 2,{ }^{13} \mathrm{E} 6,{ }^{17} \mathrm{E} 14^{25}$ \\
\hline Fadiga & $\mathrm{E} 10,{ }^{21} \mathrm{E} 25,{ }^{36} \mathrm{E} 29^{40}$ \\
\hline Raiva & $\mathrm{E} 16, \mathrm{E} 17,{ }^{27,28} \mathrm{E}^{3} 6^{47}$ \\
\hline $\begin{array}{l}\text { Algum tipo de dor física, como cefaleia, dor de } \\
\text { estômago, dor no peito }\end{array}$ & $\mathrm{E} 8,{ }^{19} \mathrm{E} 22,{ }^{33} \mathrm{E} 27^{38}$ \\
\hline Sintomas de Burnout & $\mathrm{E} 8,{ }^{19} \mathrm{E}^{3} 5^{46}$ \\
\hline Solidão & $\mathrm{E} 16,{ }^{27} \mathrm{E}^{2} 5^{46}$ \\
\hline Pânico & $\mathrm{E} 16, \mathrm{E} 17^{27,28}$ \\
\hline Perda do apetite & $\mathrm{E} 8,^{19} \mathrm{E} 32^{43}$ \\
\hline Crise de identidade profissional & $\mathrm{E} 16,{ }^{27} \mathrm{E} 28^{39}$ \\
\hline Irritabilidade & $\mathrm{E} 4,^{15} \mathrm{E} 22^{33}$ \\
\hline Pensamentos suicidas & $E 32^{43}$ \\
\hline
\end{tabular}

Fonte: Informações extraídas dos artigos científicos indexados nas bases de dados National Library of Medicine (PubMed), SCOPUS; na plataforma Web of Science, 2020 
passaram a experienciar uma pressão maior por diversos fatores que este estudo evidenciou. Considerando as profusas vantagens da realização de Scoping Review, identificou-se, de acordo com os resultados, que a China, sendo o primeiro país a sofrer os impasses da pandemia da COVID-19, tornou-se o maior produtor mundial de artigos científicos. ${ }^{52}$ No entanto, tais artigos não se propuseram ou citaram o acompanhamento dos enfermeiros e demais profissionais de saúde, ao longo do tempo, visando identificar os efeitos pós-traumáticos dessa pandemia.

Os estudos de tipo coorte foram os mais elevados nas hierarquias de evidências, na classificação dos estudos observacionais. ${ }^{53}$ No entanto, para que isso se concretize, faz-se necessário um período para análise dos fatores de risco de um dado evento. Assim, não foi possível realizar essas afirmações, visto que a COVID-19 ainda permanece como uma ameaça aos enfermeiros e aos demais profissionais de saúde - inclusive para toda a humanidade -, devido às consequências a longo prazo, e também por falta de evidências científicas de tratamento padronizado e imunização. ${ }^{54}$

Estudos de tipo coorte envolvendo profissionais que atuaram na epidemia da Síndrome Respiratória Aguda Grave (SARS) e Síndrome Respiratória do Oriente Médio (MERS) demonstraram conforme estudo encontrado nesta revisão ${ }^{44}$ - que investimentos são necessários para medidas de controle nos efeitos pós-trauma em profissionais de saúde.

O presente escopo aponta as situações que impactaram a saúde mental dos profissionais de enfermagem durante o surto da COVID-19. Essas situações estão relacionadas, principalmente, ao contexto laboral, desencadeando sinais e sintomas de sofrimento psíquico, os quais também foram evidenciados nos estudos. Entre os achados, um dos fatores mais evidentes para desencadear tal sofrimento relacionou-se à sobrecarga de trabalho. ${ }^{16,18,19,23,33,36,38,40,42,43}$

A literatura ${ }^{55}$ salienta que a equipe de enfermagem está vivenciando, em âmbito mundial, um cenário desafiador nessa pandemia, que envolve sobrecarga de trabalho associada diretamente à alta transmissibilidade do vírus e à manipulação excessiva e cuidadosa de equipamentos específicos de proteção, bem como ao excesso de horas de trabalho. ${ }^{3}$ Infere-se que tal contexto laboral pandêmico requer, desse profissional, atenção e concentração ao executar tais procedimentos, mas que essas funções mentais podem estar prejudicadas, considerando as extenuantes horas de trabalho e sensação de cansaço.

Nesse contexto, os nossos achados indicaram outros fatores laborais, por exemplo, a escassez ou ausência de Equipamentos de Proteção Individual (EPIs), ${ }^{15,23,25,27,36,38,39,48,49}$ o medo de se infectar e infectar outras pessoas, tais como os pacientes, familiares e amigos. ${ }^{12,14-17,19,21-23,33,37-39,41,46-49}$

Esses achados corroboram estudos, ${ }^{56-60}$ os quais referem que cargas extremas e longas jornadas de trabalho, esgotamento físico e mental, medo de ser infectado ou de transmitir o vírus para as pessoas próximas, realizar atividade laboral com ausência ou inadequados EPIs, baixo estoque de medicamentos e perda de amigos e familiares ${ }^{60}$ impactam a saúde mental dos profissionais de saúde. Estudo aborda que o receio dos profissionais em infectar os seus familiares ou parentes ocasiona o distanciamento e o isolamento social para protegê-los, intensificando o sofrimento mental. ${ }^{59}$

Estar na linha de frente assistindo pacientes com diagnóstico ou suspeita de COVID-19 apresenta risco de sofrimento psíquico, ${ }^{13-16,21,23,24,27,32,33,39,40,45,47}$ corroborando a literatura.$^{61}$ Estudo identificado neste manuscrito revelou que os enfermeiros hospitalares apresentaram altas taxas de sintomas de ansiedade $(60,9 \%)$ e depressão (64,8\%), ao cuidarem de pacientes diagnosticados com COVID-19, e, referente aos casos suspeitos, ansiedade $(57,7 \%)$ e de depressão (58\%), ${ }^{30}$ corroborando estudos. ${ }^{58,59}$

Outros fatores que impactaram sintomas de ansiedade e depressão relacionaram-se ao tempo de experiência ${ }^{26,27,30,47}$ e profissão exercida no serviço de saúde. ${ }^{26,27,47}$ Os estudos mostraram que, quanto mais anos de experiência o profissional de saúde tiver, menos intensos serão os sintomas de ansiedade e depressão. ${ }^{26,27,47} \mathrm{~A}$ equipe de enfermagem é a categoria profissional que está mais próxima no cuidado ao paciente com COVID-19; portanto, os riscos de infecção e de sofrerem pressão psicológica aumentam. Entretanto, o tempo de atuação e experiência no trabalho e no contexto de pandemias possibilitaram a construção de conhecimento e habilidades, que proporcionam segurança e tranquilidade emocional ao profissional de enfermagem.

Em nossos achados, os estudos $21,35,38,39,41$ demonstraram, em algum momento, que as mídias e noticiários afetaram a saúde mental dos profissionais. As Fake News são uma técnica antiga, utilizadas desde o século XX, mas que nos últimos anos, com o avanço da internet vêm se fortalecendo e possibilitando pseudoinformações ao leitor. ${ }^{62}$

Os principais sinais e sintomas de sofrimento psíquicos evidenciados neste escopo envolveram ansiedade, ${ }^{13,15,16,21-24,27-29,32,34,38-41,44-46}$ depressão, ${ }^{13,16,17,20,22-24,26,27,29,34,39,40,45,46}$ insônia, ${ }^{13,17,19,22,23,33,36,39,42,45}$ estresse, ${ }^{18,19,22,23,36,38,39,41,43,44,47}$ estresse pós-traumático, ${ }^{16,23,26,33,34,37,40,47}$ medo $^{16,21,34,40,41,43,44,46}$ e esgotamento físico e mental. ${ }^{12,19,21,34,48}$ Outros sintomas com menor frequência também foram relatados nos estudos, como angústia, ${ }^{13,17,23}$ fadiga, ${ }^{21,43,47}$ raiva, ${ }^{32,39,46}$ algum tipo de dor física - como cefaleia, dor de estômago, dor no peito ${ }^{19,37,42}$-, Burnout, ${ }^{20,34}$ solidão, ${ }^{34,46}$ pânico, ${ }^{39,46}$ diminuição de apetite $^{19,30}$ e crise de identidade profissional. ${ }^{35,46}$

Metanálise ${ }^{54}$ com o objetivo de identificar impactos na saúde física e mental de profissionais de saúde expostos à SARS, MERS e COVID-19 evidenciou sintomas psíquicos semelhantes aos nossos achados. Todavia, o sintoma comum de medo foi menor nos profissionais que atuaram na linha de frente da SARS, enquanto a insônia e a dificuldade de dormir apresentaram maiores frequências naqueles que trabalharam na linha de frente da COVID-19. Em relação ao Burnout, essa síndrome foi particularmente maior em enfermeiras que trabalharam longas horas entre pacientes com MERS. Comparando os dados de letalidade e transmissibilidade entre as doenças, tanto a SARS como a MERS constituíram-se pandemias controladas, com índices de mortalidade e transmissão na população menores 
que a COVID-19, o que poderia justificar a baixa preocupação dos profissionais de enfermagem. ${ }^{54}$

Contudo, houve altas taxas de suicídio na população em geral, no período da SARS, considerada preocupante pandemia. No entanto, quando comparada no universo dos profissionais de saúde, foram identificados casos de suicídio em trabalhadores de saúde relacionados à COVID-19. ${ }^{54}$ No que se refere ao estresse pós-traumático, percebe-se surgimento precoce no advento da COVID-19 em comparação ao da SARS e MERS. ${ }^{54}$

Diante desse contexto, cabe mencionar que várias recomendações já haviam sido feitos quanto à importância da saúde mental dos profissionais de saúde,$^{54}$ principalmente da equipe de enfermagem, ${ }^{4}$ que representa a espinha dorsal de qualquer serviço de saúde. ${ }^{63}$ Apesar de a promoção da saúde mental constituir uma das prioridades globais da agenda dos objetivos do desenvolvimento sustentável do milênio, ${ }^{64}$ pouco se investe em estratégias para identificar necessidades psicossociais e situações de vulnerabilidade emocional dos profissionais de saúde. Geralmente, a saúde física desses profissionais tem sido o principal foco de atenção, vigilância e intervenção. ${ }^{4}$

Suplementarmente a essa questão da promoção da saúde mental - apesar de não ter sido objeto de estudo deste manuscrito -, observou-se que vários estudos ${ }^{12,15-23,27,30,31,33,35,36,38-50}$ evidenciaram estratégias iniciais de apoio, sendo as primeiras relacionadas à retomada de treinamentos a fim de reduzir o medo de transmissão, ${ }^{23,30,33,36,44,47,50}$ providência de suprimentos básicos e subsídios de segurança de trabalho para a equipe de linha de frente. ${ }^{21,43,47,50}$

Concomitantemente, houve também recomendações ou utilização de estratégias de apoio, visando prevenir situações de sofrimento psíquico, sendo a de maior aderência a disponibilização de equipes para suporte psicossocial e técnico de intervenção psicológica, ${ }^{15,18-26,30,33,34,39,43-45,47}$ bem como assistência de psicoeducação por telefone e WebChat. ${ }^{15,19,30,38,39}$

Outras estratégias envolveram a valorização do apoio relacional e comunicacional com colegas de trabalho ou por pares, ${ }^{12,19,23,30,33,38,44}$ tais como bate-papo por vídeo ou telefone com os membros da família, ${ }^{19,47}$ incentivo à realização de atividades esportivas de canto, de redação de diários, exibição de vídeos, alimentação e atendimento de necessidades físicas básicas. ${ }^{19-21,30,38,43,47}$ No âmbito laboral dos profissionais, houve melhorias no espaço de descansos. ${ }^{15,20,38,43,44}$ Três estudos ${ }^{18,33,35}$ ofereceram incentivo financeiro como estratégia de apoio, e apenas um relatou redução da carga horária de trabalho. ${ }^{40}$

Outra estratégia de suporte relevante consistiu no aprimoramento do grupo de assistência para fortalecer o atendimento humanitário, constituído por líderes de equipe e colegas de trabalho, com a missão de visitarem regularmente os familiares das equipes de enfermagem, a fim de identificar e resolver suas solicitudes em tempo hábil, uma vez que os profissionais relatavam grande preocupação com eles. ${ }^{30} \mathrm{O}$ envolvimento dos líderes de equipe, comunicação e bom relacionamento com gestores e diversos líderes hospitalares também foram temas mencionados em outros estudos desse escopo. ${ }^{35,44}$ Tal estratégia tem sido utilizada com o objetivo de melhorar o ambiente de trabalho, visto que o diálogo efetivo entre chefias e equipes, por meio de reuniões, possibilita perceber e identificar precocemente sinais e sintomas que possam retratar qualquer situação de risco. ${ }^{4}$ No entanto, é preciso frisar - assim como nos estudos encontrados ${ }^{44,46}$ - que todas essas estratégias mencionadas acima não deveriam ser utilizadas apenas em contexto de emergência de grande porte. É preciso dar continuidade permanente a fim de garantir, a longo prazo, mecanismos de resiliência e apoio à equipe de enfermagem e todos os outros profissionais de saúde, considerando que as emergências do tipo pandêmica serão constantes. ${ }^{54}$

Particularmente no Brasil, a maioria dos serviços de saúde não dispõe de experiências de atuação em emergências de grande porte - como é o caso da COVID-19 -, o que representa um estressor adicional para as equipes. ${ }^{6}$ Assim, é preciso que sejam estruturadas, com veemência, estratégias de apoio a essa categoria de trabalhadores, uma vez que a saúde mental, além de fazer parte dos objetivos do desenvolvimento sustentável do milênio, ${ }^{64}$ também se articula com a meta da Agenda 2030, que prevê a necessidade de nove milhões de enfermeiros e parteiras para conseguir atingir uma cobertura universal de saúde. ${ }^{63}$

Antes do advento da pandemia da COVID-19, no Brasil, a equipe de enfermagem já apresentava sinais de esgotamento, pois a literatura constatou que cerca de $56 \%$ desses profissionais adoeceram nos últimos 12 meses, acidentaram-se no trabalho, e aproximadamente 180 mil profissionais, no último ano, se sentiam desassistidos. ${ }^{65}$

\section{CONCLUSÃO E IMPLICAÇÕES PARA A PRÁTICA}

O estudo evidenciou que os profissionais de enfermagem enfrentam situações de sofrimento psíquico, principalmente desencadeadas por fatores relacionados às condições de trabalho, manifestando sintomas depressivos, de ansiedade e de estresse, que podem permanecer por longo período de tempo. Os resultados também salientaram que as instituições de saúde precisaram implementar ações de capacitação, proteção e segurança, bem como suporte e apoio psicossocial em curto espaço de tempo. Contudo, tais estratégias necessitam ser permanentes, o que requer investimento financeiro contínuo, monitoramento da saúde mental desses profissionais por longo prazo e tratamento pelas instituições de saúde públicas e privadas. Esse escopo identificou que os estudos abordaram contexto hospitalar, o que demonstra a lacuna de produção de conhecimento na rede de atenção primária à saúde e préhospitalar. Ademais, a produção científica não foi identificada em alguns continentes, denotando, assim, a necessidade de atitudes sociais e políticas públicas em torno da saúde mental desses profissionais.

Vale mencionar que a ausência de fomento para a realização desta pesquisa restringiu o acesso a artigos na íntegra mediante pagamento, consolidando-se como uma limitação. 


\section{FINANCIAMENTO}

O presente trabalho foi realizado com apoio da Coordenação de Aperfeiçoamento de Pessoal de Nível Superior - Brasil (CAPES) - Código de Financiamento 001, bolsa de Mestrado, concedida a Caroline dos Santos Pereira e bolsa de Doutorado, concedida a Sarah Salvador Pereira.

\section{CONTRIBUIÇÕES DOS AUTORES}

Desenho do estudo. Mellina Yamamura. Simone Teresinha Protti-Zanatta. Sonia Regina Zerbetto. Fernanda Berchelli Girão Miranda.

Coleta ou produção dos dados. Sarah Salvador Pereira. Caroline dos Santos Pereira. Marceli Costa. Fernanda Berchelli Girão Miranda.

Análise de dados. Sarah Salvador Pereira. Caroline dos Santos Pereira. Marceli Costa. Fernanda Berchelli Girão Miranda. Interpretação dos resultados. Sarah Salvador Pereira. Caroline dos Santos Pereira. Marceli Costa. Fernanda Berchelli Girão Miranda.

Redação e revisão crítica do manuscrito. Mellina Yamamura. Sarah Salvador Pereira. Caroline dos Santos Pereira. Simone Teresinha Protti-Zanatta. Sonia Regina Zerbetto. Marceli Costa. Aprovação da versão final do artigo. Mellina Yamamura. Sarah Salvador Pereira. Caroline dos Santos Pereira. Simone Teresinha Protti-Zanatta. Sonia Regina Zerbetto. Marceli Costa. Fernanda Berchelli Girão Miranda.

Responsabilidade por todos os aspectos do conteúdo e a integridade do artigo publicado. Mellina Yamamura. Sarah Salvador Pereira. Caroline dos Santos Pereira. Simone Teresinha Protti-Zanatta. Sonia Regina Zerbetto. Marceli Costa. Fernanda Berchelli Girão Miranda.

\section{EDITOR ASSOCIADO}

Cristina Rosa Baixinho

\section{REFERÊNCIAS}

1. Singhal T. A review of Coronavirus Disease-2019 (COVID-19). Indian J Pediatr. 2020;87(4):281-6. http://dx.doi.org/10.1007/s12098-02003263-6. PMid:32166607.

2. World Health Organization. Situation reports. [Internet]. WHO; 2020 [citado 2020 Mar]. Disponível em: https://www.who.int/emergencies/ diseases/novel-coronavirus-2019/situation-reports.

3. Torales J, O'Higgins M, Castaldelli-Maia JM, Ventriglio A. The outbreak of COVID-19 coronavirus and its impact on global menta health. Int J Soc Psychiatry. 2020 jun;66(4):317-20. http://dx.doi. org/10.1177/0020764020915212. PMid:32233719

4. Oliveira WA, Oliveira-Cardoso EA, Silva JL, Santos MA. Impactos psicológicos e ocupacionais das sucessivas ondas recentes de pandemias em profissionais da saúde: revisão integrativa e lições aprendidas. Estud Psicol. 2020;37:e200066. http://dx.doi.org/10.1590/19820275202037 e200066.

5. Jiang X, Deng L, Zhu Y, Ji H, Tao L, Liu L et al. Psychological crisis intervention during the outbreak period of new coronavirus pneumonia from experience in Shanghai. Psychiatry Res. 2020;286:1-3. http:// dx.doi.org/10.1016/j.psychres.2020.112903. PMid:32146245.
6. Marques LC, Lucca DC, Alves EO, Fernandes GCM, Nascimento KC COVID-19: cuidados de enfermagem para segurança no atendimento de serviço pré-hospitalar móvel. Texto Contexto Enferm. 2020;29:1-12. http://dx.doi.org/10.1590/1980-265x-tce-2020-0119.

7. Rotelli F, Leonardis O, Mauri D, Risio C. Desinstitucionalizaçao. São Paulo: Hucitec, 1990.

8. Peters MDJ, Godfrey C, Mclnerney P, Baldini SC, Khalil H, Parker D. Chapter 11: Scoping Reviews. In: Aromataris E, Munn Z, editors. Joanna Briggs Institute Reviewer's Manual. [Internet]. JBI; 2017. [citado 2020 Mar]. Disponível em: https://reviewersmanual.joannabriggs.org/.

9. Tricco AC, Lillie E, Zarin W, O'Brien KK, Colquhoun H, Levac D et al. PRISMA extension for scoping reviews (PRISMA-ScR): checklist and explanation. Ann Intern Med. 2018;169(7):467-73. http://dx.doi org/10.7326/M18-0850. PMid:30178033.

10. Munn Z, Peters MDJ, Stern C, Tufanaru C, McArthur A, Aromataris E. Systematic review or scoping review? Guidance for authors when choosing between a systematic or scoping review approach. BMC Med Res Methodol.2018;18(1):143. http://dx.doi.org/10.1186/s12874-0180611-x. PMid:30453902

11. Tricco AC, Lillie E, Zarin W, O'Brien K, Colquhoun H, Kastner M et al. A scoping review on the conduct and reporting of scoping reviews. BMC Med Res Methodol. 2016;16(15):1-10. http://dx.doi.org/10.1186/ s12874-016-0116-4. PMid:26857112.

12. Xiang YT, Jin Y, Wang Y, Zhang Q, Zhang L, Cheung T. Tribute to health workers in China: A group of respectable population during the outbreak of the COVID-19. Int J Biol Sci. 2020;16(10):1739-40. http://dx.doi. org/10.7150/ijbs.45135. PMid:32226292.

13. Lai J, Ma S, Wang Y, Cai Z, Hu J, Wei N et al. Factors associated with mental health outcomes among health care workers exposed to coronavirus disease 2019. JAMA Netw Open. 2020;3(3):e203976. http:// dx.doi.org/10.1001/jamanetworkopen.2020.3976. PMid:32202646.

14. Li Z, Ge J, Yang M, Feng J, Qiao M, Jiang R et al. Vicarious traumatization in the general public, members, and non-members of medical teams aiding in COVID-19 control. Brain Behav Immun. 2020, S08891591(20):30309-3. https://doi.org/10.1016/j.bbi.2020.03.007.

15. Chen Q, Liang M, LiY, Guo J, Fei D, Wang L et al. Mental health care for medical staff in China during the COVID-19 outbreak. Lancet Psychiatry $2020 \mathrm{abr} ; 7(4): e 15-6$. http://dx.doi.org/10.1016/S2215-0366(20)30078-X. PMid:32085839.

16. Xiang YT, Yang Y, Li W, Zhang L, Zhang Q, Cheung T et al. Timely mental health care for the 2019 novel coronavirus outbreak is urgently needed. Lancet Psychiatry. 2020;7(3):228-9. http://dx.doi.org/10.1016/ S2215-0366(20)30046-8. PMid:32032543.

17. Kang L, Ma S, Chen M, Yang J, Wang Y, Li R et al. Impact on Menta Health and Perceptions of Psychological Care among Medical and Nursing Staff in Wuhan during the 2019 Novel Coronavirus Disease Outbreak: a Cross-sectional Study. Brain Behav Immun. 2020;87:11-7. http://dx.doi.org/10.1016/j.bbi.2020.03.028. PMid:32240764.

18. Mo Y, Deng L, Zhang L, Lang Q, Liao C, Wang N et al. Work stress among Chinese nurses to support Wuhan in fighting against COVID-19 epidemic. J Nurs Manag. 2020;28(5):1002-9. http://dx.doi.org/10.1111/ jonm.13014. PMid:32255222.

19. Cao J, Wei J, Zhu H, Duan Y, Geng W, Hong X et al. A Study of Basic Needs and Psychological Wellbeing of Medical Workers in the Fever Clinic of a Tertiary General Hospital in Beijing during the COVID-19 Outbreak. Psychother Psychosom. 2020;89(4):252-4. http://dx.doi. org/10.1159/000507453. PMid:32224612.

20. Liang Y, Chen M, Zheng X, Liu J. Screening for Chinese medical staff mental health by SDS and SAS during the outbreak of COVID-19. J Psychosom Res. 2020;133:110102. http://dx.doi.org/10.1016/j. jpsychores.2020.110102. PMid:32224344.

21. Sun T, Wei L, Shi S, Jiao D, Song R, Ma L et al. A qualitative study on the psychological experience of caregivers of COVID-19 patients Am J Infect Control. 2020;48(6):592-8. http://dx.doi.org/10.1016/j. ajic.2020.03.018. PMid:32334904.

22. Zhang C, Yang L, Liu S, Ma S, Wang Y, Cai Z et al. Survey of insomnia and related social psychological factors among medical staff involved with the 2019 novel coronavirus disease outbreak. Front Psychiatry. 
2020;11(306):306. http://dx.doi.org/10.3389/fpsyt.2020.00306. PMid:32346373.

23. Wang S, Wen X, Liu B, Dong Y, Cui M. Psychological influence of Coronavirus disease 2019 (COVID-19) pandemic on the general public, medical workers and patients with mental disorders and its countermeasures. Psychosomatics. 2020;61(6):616-24. http://dx.doi. org/10.1016/j.psym.2020.05.005. PMid:32739051.

24. Ni MY, Yang L, Leung CMC, Li N, Yao XI, Wang Y et al. Mental Health, risk factors, and social media use during the COVID-19 epidemic and cordon sanitaire among the community and health professionals in Wuhan, China: cross-sectional survey. JMIR Ment Health. 2020;7(5):e19009. http://dx.doi.org/10.2196/19009. PMid:32365044.

25. Liu CY, Yang YZ, Zhang XM, Xu X, Dou QL, Zhang WW et al. The prevalence and influencing factors in anxiety in medical workers fighting COVID-19 in China: A cross-sectional survey. Epidemiol Infect. 2020;148(98):1-7. http://dx.doi.org/10.1017/S0950268820001107. PMid:32430088.

26. Song X, Fu W, Xiaoran L, Liu X, Luo Z, Wang R et al. Mental health status of medical staff in emergency departments during the Coronavirus disease 2019 epidemic in China. Brain Behav Immun. Print. 2020;88;6065. https://doi.org/10.1016/j.bbi.2020.06.002

27. Xiao X, Zhu X, Fu S, HuY, Li X, Xiao J. Psychological impact of healthcare workers in China during COVID-19 pneumonia epidemic: a multi-center cross-sectional survey investigation. J Affect Disord. 2020;274:405-10. http://dx.doi.org/10.1016/j.jad.2020.05.081. PMid:32663970.

28. Cai W, Lian B, Song X, Hou T, Deng G, Li H. A cross-sectional study on mental health among health care workers during the outbreak of Corona Virus Disease 2019. Asian J Psychiatr. 2020;51:102111. http:// dx.doi.org/10.1016/j.ajp.2020.102111. PMid:32361388.

29. Juhong Z, Lin S, Lan Z, Wang H, Fan A, Yang B et al. Prevalence and influencing factors of anxiety and depression symptoms in the first-line medical staff fighting against COVID-19 in Gansu. Front Psychiatry. 2020;11:386. http://dx.doi.org/10.3389/fpsyt.2020.00386. PMid:32411034.

30. Shen X, Zou X, Zhong X, Yan J, Li L. Psychological stress of ICU nurses in the time of COVID-19. Crit Care. 2020;24(1):200. http://dx.doi. org/10.1186/s13054-020-02926-2. PMid:32375848.

31. Sun D, Yang D, LiY, Zhou J, Wang W, Wang Q et al. Psychological impact of 2019 novel coronavirus (2019-nCoV) outbreak in health workers in China. Epidemiol Infect. 2020;148:e96. http://dx.doi.org/10.1017/ S0950268820001090. PMid:32430086.

32. Cai H, Tu B, Ma J, Chen L, Fu L, Jiang Y et al. Psychological Impact and Coping Strategies of Frontline Medical Staff in Human Between January and March 2020 During the Outbreak of Coronavirus Disease 2019 (COVID-19) in Hubei, China. Med Sci Monit. 2020;26:e924171. http://dx.doi.org/10.12659/MSM.924171. PMid:32291383.

33. Conversano C, Marchi L, Miniati M. Psychological distress among healthcare professionals involved in the Covid-19 emergency: vulnerability and resilience factors. Clinical Neuropsychiatry. 2020;17(2):94-6. http:// dx.doi.org/10.36131/CN20200212.

34. Cheung T, Fong TKH, Bressington D. COVID-19 under the SARS Cloud: Mental Health Nursing during the Pandemic in Hong Kong. J Psychiatr Ment Health Nurs. 2020;1-3:jpm.12639. http://dx.doi.org/10.1111/ jpm.12639. PMid:32311811.

35. Fawaz M, Samaha A. The psychosocial effects of being quarantined following exposure to COVID-19: A qualitative study of Lebanese health care workers. Int J Soc Psychiatry. 2020;66(6):1-6. http://dx.doi. org/10.1177/0020764020932202. PMid:32489149.

36. Tan BYQ, Chew NWS, Lee GKH, Jing M, Goh Y, Yeo LLL et al. Psychological Impact of the COVID-19 Pandemic on Health Care Workers in Singapore. Ann Intern Med. 2020;173(4):317-20. http://dx.doi.org/10.7326/M201083. PMid:32251513.

37. Nicholas WS, Chewa GKH, Leeb BYQ, Tanb C, Mingxue J, Yihui G et al. A multinational, multicentre study on the psychological outcomes and associated physical symptoms amongst healthcare workers during COVID19 outbreak. Brain Behav Immun. 2020;88:559-565. http://dx.doi. org/10.1016/j.bbi.2020.04.049.

38. Rajkumar RP. COVID-19 and mental health: A review of the existing literature. Asian J Psychiatr. 2020;52:102066. http://dx.doi.org/10.1016/j. ajp.2020.102066. PMid:32302935.
39. Grover S, Dua D, Sahoo S, Mehra A, Nehra R, Chakrabarti S. Why all COVID-19 hospitals should have mental health professionals: The importance of mental health in a worldwide crisis! Asian J Psychiatr. 2020;51:102147. http://dx.doi.org/10.1016/j.ajp.2020.102147. PMid:32473537.

40. Mukhtar S. Mental Well-Being of Nursing Staff During the Coronavirus Disease 2019 Outbreak: A Cultural Perspective. J Emerg Nurs. 2020;1 2(4):426-7. http://dx.doi.org/10.1016/j.jen.2020.04.003. PMid:32418672.

41. Temsah MH, Al-Sohime F, Alamro N, Al-Eyadhy A, Al-Hasan K, Jamal $A$ et al. The psychological impact of COVID-19 pandemic on health care workers in a MERS-CoV endemic country. J Infect Public Health. 2020;13(6):877-82. http://dx.doi.org/10.1016/j.jiph.2020.05.021. PMid:32505461.

42. Barello S, Palamenghi L, Graffigna G. Burnout and somatic symptoms among frontline healthcare professionals at the peak of the Italian COVID-19 pandemic. Psychiatry Res. 2020;290:1-4. http://dx.doi. org/10.1016/j.psychres.2020.113129. PMid:32485487.

43. Blanco-Donoso LM, Garrosa E, Moreno-Jiménez J, Gálvez-Herrer $\mathrm{M}$, Moreno-Jiménez B. Occupational psychosocial risks of health professionals in the face of the crisis produced by the COVID-19: From the identification of these risks to immediate action. Int J Nurs Stud. 2020;2:100003. http://dx.doi.org/10.1016/j.jinsa.2020.100003.

44. Maben J, Bridges J. Covid-19: Supporting nurses' psychological and mental health. J Clin Nurs. 2020;29(15-16):2742-50. http://dx.doi. org/10.1111/jocn.15307. PMid:32320509.

45. Pappa S, Ntella V, Giannakas T, Giannakoulis VG, Papoutsi E, Katsaounou P. Prevalence of depression, anxiety, and insomnia among healthcare workers during the COVID-19 pandemic: A systematic review and meta-analysis. Brain Behav Immun. 2020;S0889-1591(20):30845-X. http://dx.doi.org/10.1016/j.bbi.2020.05.026.

46. Albott CS, Wozniak JR, McGlinch BP, Wall MH, Gold BS, Vinogradov S. Battle buddies: rapid deployment of a psychological resilience intervention for health care workers during the COVID-19 Pandemic. Anesth Analg. 2020;131(1):43-54. http://dx.doi.org/10.1213/ANE.0000000000004912. PMid:32345861.

47. Kisely S, Warren N, McMahon L, Dalais C, Henry I, Siskind D. Occurrence, prevention, and management of the psychological effects of emerging virus outbreaks on healthcare workers: rapid review and meta-analysis. BMJ. 2020;369:m1642. http://dx.doi.org/10.1136/bmj. m1642. PMid:32371466.

48. Alharbi J, Jackson D, Usher K. The potential for COVID-19 to contribute to compassion fatigue in critical care nurses. J Clin Nurs. 2020;29(1516):2762-4. http://dx.doi.org/10.1111/jocn.15314. PMid:32344460.

49. Spoorthy MS, Pratapa SK, Mahant S. Mental health problems faced by healthcare workers due to the COVID-19 pandemic-A. Asian J Psychiatr.2020;51:102119. http://dx.doi.org/10.1016/j.ajp.2020.102119. PMid:32339895.

50. Humerez DC, Ohl RIB, Silva MCN. Mental health of Brazilian nursing professionals in the context of the covid-19 pandemic: action of the Nursing Federal Council. Cogitare enferm. 2020; 25:1-10. http://dx.doi. org/10.5380/ce.v25i0.74115.

51. Catton H. Global challenges in the health and health care for nurses and midwives everywhere. Int Nurs Rev. 2020;67(1):4-6. http://dx.doi. org/10.1111/inr.12578. PMid:32083728.

52. Tollefson J. China declared world's largest producer of scientific articles. Nature. 2018;553(7689):390. http://dx.doi.org/10.1038/d41586-01800927-4.

53. Thiese MS. Observational and interventional study design types; an overview. Biochem Med (Zagreb). 2014;24(2):199-210. http://dx.doi. org/10.11613/BM.2014.022. PMid:24969913.

54. Salazar de Pablo G, Vaquerizo-Serrano J, Catalan A, Arango C, Moreno $\mathrm{C}$, Ferre $\mathrm{F}$ et al. Impact of coronavirus syndromes on physical and mental health of health care workers: Systematic review and metaanalysis. J Affect Disord. 2020;275:48-57. http://dx.doi.org/10.1016/j. jad.2020.06.022. PMid:32658823.

55. Miranda FMA, Santana LL, Pizzolato AC, Saquis LMM. Working conditions and the impact on the health of the nursing professionals in the context of Covid-19. Cogitare enferm. 2020;25. http://dx.doi. org/10.5380/ce.v25i0.72702. 
Sofrimento psíquico, enfermagem e pandemia da COVID-19

Miranda FBG, Yamamura M, Pereira SS, Pereira CS, Protti-Zanatta ST, Costa MK, Zerbetto SR

56. Santos CF. Reflections about the impact of the SARS-COV-2/COVID-19 pandemic on mental health. Br J Psychiatry. 2020;42(3):329. http:// dx.doi.org/10.1590/1516-4446-2020-0981. PMid:32321063.

57. The Lancet. The Lancet. COVID-19: protecting health-care workers. Lancet. 2020;395(10228):922. http://dx.doi.org/10.1016/S01406736(20)30644-9.

58. Walton M, Murray E, Christian MD. Mental health care for medical staff and affiliated healthcare workers during the COVID-19 pandemic. Eur Heart J Acute Cardiovasc Care. 2020;9(3):241-7. http://dx.doi. org/10.1177/2048872620922795. PMid:32342698.

59. Liu Q, Luo D, Haase JE, Guo Q, Wang XQ, Liu S et al. The experiences of health-care providers during the COVID-19 crisis in China: a qualitative study. Lancet Glob Health. 2020;8(6):e790-8. http://dx.doi.org/10.1016/ S2214-109X(20)30204-7. PMid:32573443.

60. Prado AD, Peixoto BC, Silva AMB, Scalia LAM. A saúde mental dos profissionais de saúde frente à pandemia do COVID-19: uma revisão integrativa. REAS jun 2020;46:e4128. https://doi.org/10.25248/reas.e4128.2020.
61. Arpacioglu S, Gurler M, Cakiroglu S. Secondary Traumatization Outcomes and Associated Factors Among the Health Care Workers Exposed to the COVID-19. Int J Soc Psychiatry. 2020;00(0):1-6. http:// dx.doi.org/10.1177/0020764020940742. PMid:32635786.

62. Neto M, Gomes TO, Porto FR, Rafael RMR, Fonseca MHS, Nascimento J. Fake news in the context of the covid-19 pandemic. Cogitare enferm. 2020;25. http://dx.doi.org/10.5380/ce.v25i0.72627.

63. World Health Organization. Director-General's opening remarks at the media briefing on COVID-19. Genebra: WHO; 2020

64. Orellana JDY, Ribeiro MRC, Barbieri MA, Saraiva MC, Cardoso VC, Bettiol $\mathrm{H}$ et al. Mental disorders in adolescents, youth, and adults in the RPS Birth Cohort Consortium (Ribeirão Preto, Pelotas and São Luís), Brazil. Cad Saude Publica. 2020;36(2):e00154319. http://dx.doi. org/10.1590/0102-311x00154319. PMid:32022176.

65. Silva, MCN, Machado MH. Health and Work System: challenges for the Nursing in Brazil. Ciênc. saúde coletiva. 2020 jan;25(1):7-13. https:// doi.org/10.1590/1413-81232020251.27572019. 